\title{
Transition Metal-based Anticancer Drugs Targeting Nucleic Acids: A Tandem Mass Spectrometric Investigation
}

\author{
Rahel P. Eberle, Yvonne Hari, and Stefan Schürch*
}

\begin{abstract}
The search for effective drugs against cisplatin-resistant tumors resulted in a large number of organometallic compounds that are evaluated for their antiproliferative activity. Among the most promising candidates are bent metallocenes based on various transition metal ions and ligands. The elucidation of structural features and the characterization of the interaction of a drug candidate with its target require accurate and sensitive analytical tools. Tandem mass spectrometry is applied to the investigation of the adduct sites and binding patterns of metallodrugs bound to single-stranded oligonucleotides and higher-order nucleic acids. Results reveal the binding specificities of the different metallodrugs and demonstrate the influence they exert on the dissociation pathways of the adducts in the gas-phase.
\end{abstract}

Keywords: Anticancer drugs · Cisplatin · Metallocenes · Nucleic acids · Tandem mass spectrometry

\section{Introduction}

Research in the life sciences has been strongly accelerated over the past two decades by the availability of mass spectrometry-based techniques for the analysis of peptide, protein, and lipid biomarkers, as well as drugs and their metabolites. Owing to its high selectivity and sensitivity, tandem mass spectrometry has evolved into one of the most powerful tools for the elucidation of molecular structures, the sequencing of biopolymers, and the comprehensive quantitation of organic compounds in complex matrices. The characterization of complex mixtures can be approached by a combination of chromatographic separation, soft ionization, and highresolution mass analysis. Furthermore, the ability of tandem mass spectrometry (MS/MS) to select and activate precursor ions in the gas-phase makes the method inherently well suited for the analysis of heterogeneous samples. In contrast to the rapid expansion of mass spectrometry-

\footnotetext{
${ }^{*}$ Correspondence: Prof. Dr. S. Schürch Department of Chemistry and Biochemistry University of Bern

Freiestrasse 3

$\mathrm{CH}-3012$ Bern

E-mail: stefan.schuerch@dcb.unibe.ch
}

based proteomics and diagnostics, the characterization of nucleic acids remains a niche application. Nevertheless, the identification and quantitation of nucleic acidmodifications, the sequencing of highly modified therapeutic oligonucleotides, and the elucidation of drug-nucleic acid interactions represent key applications that benefit from the strengths of tandem mass spectrometry.

\section{Sequencing of Nucleic Acids by Tandem Mass Spectrometry}

When seen from a distance, the tandem mass spectrometric sequencing of any biopolymer might essentially follow the same principle. Analyte ions of interest are isolated in the gas-phase and their internal energy is increased, commonly by collision with an inert gas in a process referred to as collision-induced dissociation (CID). As a result of this activation, covalent bonds are cleaved and one or more series of backbone fragments are generated, which provide the desired sequence information. However, due to unique structural features, peptides and oligonucleotides exhibit completely different gas-phase behaviors. While peptides predominantly dissociate by scission of the amide bond that links two adjoining amino acids, the structure dependence of oligonucleotide fragmentation is much more pronounced and more often than not, several dissociation events coincide. The backbone cleavage of deoxyribonucleic acids (DNA) is always accompanied by the loss of a nucleobase, as this represents the initial step of the dissociation mechanism eventually resulting in the cleavage of the 3 '-C-O bond and the formation of the so-called (a-B)- and w-fragment ions (Fig. 1).[1] In contrast, the cleavage of the 5 '-P-O phosphoester bond in ribonucleic acids (RNA) is independent of nucleobase loss and results in the c- and y-fragment ions. ${ }^{[2,3]}$ Oligonucleotides administered for therapeutic purposes (e.g. for antisense applications) commonly comprise structural modifications of the backbone, the sugar moiety, or the nucleobases in order to improve their biostability and to tailor their interaction with the complementary nucleic acid targets. Such modifications influence the dissociation pathways of the oligonucleotides and, consequently, affect the resulting product ion spectra. ${ }^{[4-6]}$ In any case, charge-induced nucleobase loss, the occurrence of internal fragments due to secondary dissociation events, and the presence of ubiquitous alkali ion adducts further complicate the mass spectral data.

\section{Software-supported Spectra Interpretation}

The great diversity of possible dissociation products turns the elucidation of nucleotide sequences and their structural modifications into a very timeconsuming and error-prone process. Spectra interpretation is greatly facilitated by the software tool OMA \& OPA (oligonucleotide mass assembler \& oligonucleotide peak analyzer) developed in our department. ${ }^{[7]}$ The Java-based software calculates the masses of all theoretically possible fragments of a given single-stranded oligonucleotide or duplex 


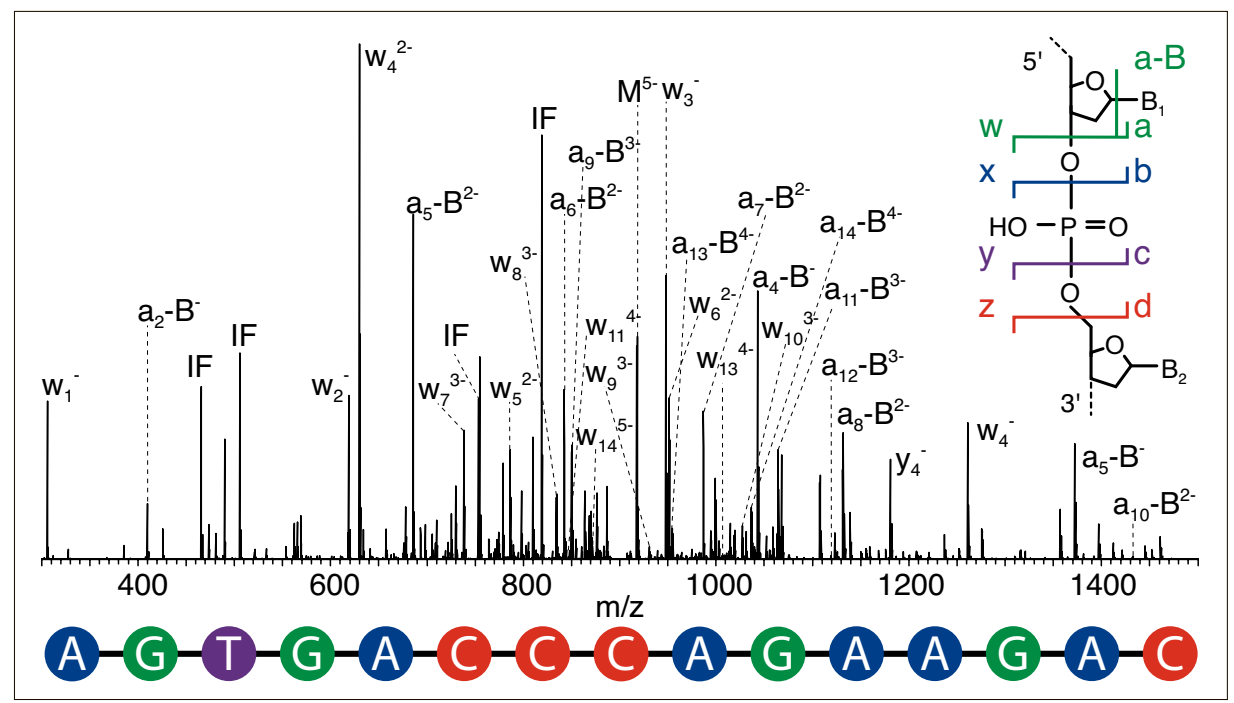

Fig. 1. Product ion spectrum generated by collisional activation of a fivefold charged DNA $15 \mathrm{mer}$. The nucleotide sequence is reflected by the DNA-typical (a-B)- and $w$-fragment ion series produced by cleavage of the 3 '-C-O bond. Alternative dissociation channels and secondary fragmentation events give rise to additional ion series and internal fragment ions (IF).

sequence and matches the complete mass list with the product ion spectrum. Userdefined structural modification of the input sequence as well as adducts with any cation or anion can easily be implemented, which makes the tool extremely helpful for the characterization of nucleic acidmetallodrug adducts. The OMA \& OPA software can be downloaded from http://schuerch.dcb.unibe.ch/index.php/ research-projects/oma-opa-software.

\section{Characterization of Nucleic Acid- Metallodrug Interactions}

\section{Cisplatin Adducts}

Cisplatin (cis-diamminedichloroplatinum (II)) has been a strong pillar of anticancer chemotherapy for decades and is still administered today, mostly in combination with newer-generation cytotoxic agents. The highly reactive cisplatin forms covalent intra- and interstrand cross-links in the DNA double helix. The thereby caused structural alterations, such as local unwinding and the bending of the double helix, interfere with replication and transcription processes and thus are responsible for the antiproliferative effect of cisplatin. Though cisplatin is highly effective against various types of tumors, patients often suffer from severe side effects and the treatment may be unsuccessful due to intrinsic or acquired resistance of cancer cells against drug-induced apoptosis. These shortcomings encouraged the development of novel metallodrugs based on platinum and alternative transition metals.

The quest for effective and less toxic anticancer drugs demands powerful analytical techniques to characterize the in- teraction of drug candidates with their cellular targets. Various analytical techniques, including NMR, X-ray diffraction, and mass spectrometry, have been applied for that purpose. The latter can give rapid proof for the interaction between metallodrugs and their nucleic acid targets and provide information about the stoichiometry of the complexes. Examples thereof range from established platinum-based anticancer drugs to current metallodrug candidates based on various transition metals. ${ }^{[8-10]}$ Since adduct formation represents a structural alteration that influences the accessibility of dissociation channels, the spectra resulting from tandem mass spectrometric experiments reflect the adduct sites and binding patterns. The effect of adduct formation on the gas-phase decomposition has been demonstrated for cisplatin bound to single-stranded model oligonucleotides ${ }^{[5,11,12]}$ as well as higherorder structures. ${ }^{[13]}$ Platination, which primarily occurs at GG and AG base pairs, was found to weaken the $\mathrm{N}$-glycosidic bond and, consequently, to promote backbone cleavage on the 3'-side of the adduct, resulting in the corresponding w-fragment ion.

\section{Metallocene Adducts}

Among the metallodrugs evaluated for their antiproliferative potential against cancer cells, bent metallocenes gained increased attention already in the 1990s. Initial expectations on titanocene dichloride $\left(\mathrm{Cp}_{2} \mathrm{TiCl}_{2}\right)$ shattered due to a lack of effectiveness against breast and renal cell carcinomas and the candidate was not pursued beyond phase II clinical trials. ${ }^{[14,15]}$ Nevertheless, bent metallocenes comprising functionalized cyclopentadienyl ligands, such as titanocene Y, which exhibits methoxybenzylsubstituted ligands, moved into the focus of anticancer drug development. ${ }^{[16]}$

In contrast to planar platinum-based drugs, the binding preferences and binding patterns of bent metallocenes to single- and double-stranded nucleic acids have notbeen elucidated in detail yet. Since metallodrugs do not only target a single, well-defined site in nucleic acids, heterogeneous mixtures of adducts are generated upon incubation with oligonucleotides, which renders their characterization difficult. Despite the increased sample complexity, adducts exhibiting different stoichiometries can easily be separated in the gas-phase and selected as precursors for tandem mass spectrometric analysis.

Initial experiments on simple model systems revealed very diverse target specificities and binding patterns of metallocenes based on different transition metal ions. The binding of metallocenes to deoxydinucleoside monophosphates was found to follow the Pearson hardsoft acid-base (HSAB) concept. Metal ions with small radii and high charge (e.g. $\left.\mathrm{Ti}^{4+}\right)$ are classified as hard Lewis acids that preferentially bind to hard Lewis bases, such as the oxygen atoms of phosphate groups. This preference is reflected by the product ion spectra of the adducts formed between titanocene and the dinucleoside monophosphates $\mathrm{d}(\mathrm{CpT})$ and $\mathrm{d}(\mathrm{TpC})$. In Fig. 2a, the base peak $(\mathrm{m} / \mathrm{z} 499)$ refers to the $\left[\mathrm{w}_{1}+\mathrm{Cp}_{2} \mathrm{Ti}\right]^{+}$fragment ion, which is generated by the scission of the $3^{\prime}-\mathrm{C}-\mathrm{O}$ bond. The peak at $\mathrm{m} / \mathrm{z} 355$ corresponds to $\left[\mathrm{w}_{1}+\mathrm{Cp}_{2} \mathrm{Ti}-\mathrm{TH}-\mathrm{H}_{2} \mathrm{O}\right]^{+}$(structure shown in Fig. 2), a secondary fragment ion that has lost its nucleobase and a water molecule. The presence of this secondary fragment ion points to a strong interaction of titanium with the phosphate group. Due to the lack of a second phosphate moiety in dinucleoside monophosphates, the nucleobase represents the alternative binding site. Such binding was found to be highly nucleobase specific with a preferred binding to thymine. This is evidenced by the $\left[\mathrm{w}_{1}+\mathrm{Cp}_{2} \mathrm{Ti}\right]^{+}$ion derived from the d(CpT) adduct (Fig. 2a), while $\left[\mathrm{M}+\mathrm{Cp}_{2} \mathrm{Ti}-\mathrm{CH}-\mathrm{H}_{2} \mathrm{O}\right]^{+}$constitutes the main dissociation product of the adduct with reversed base order (Fig. 2b).

On the other hand, larger, more polarizable metal ions (e.g. $\mathrm{Mo}^{4+}$ or $\left.\mathrm{Pt}^{2+}\right)$ constitute softer Lewis acids that target soft bases, such as electron-rich nitrogen atoms of the nucleobases. This preference is evidenced by the highly abundant peak of the $\left[\mathrm{G}+\mathrm{Cp}_{2} \mathrm{Mo}\right]^{+}$fragment in the product ion spectrum of the $\mathrm{d}(\mathrm{ApG})$ molybdenocene adduct (Fig. 2c). The spectrum also reveals that the bond to the 
Fig. 2. Product ion spectra generated by collisional activation $d(C p T)$ and $b) d(T p C)$ with titanocene and c) d(ApG) with molybdenocene. In the applied nomenclature, $G$ corresponds to a negatively charged guanine, whereas $\mathrm{GH}$ indicates a neutral nucleobase. of the adducts of a)

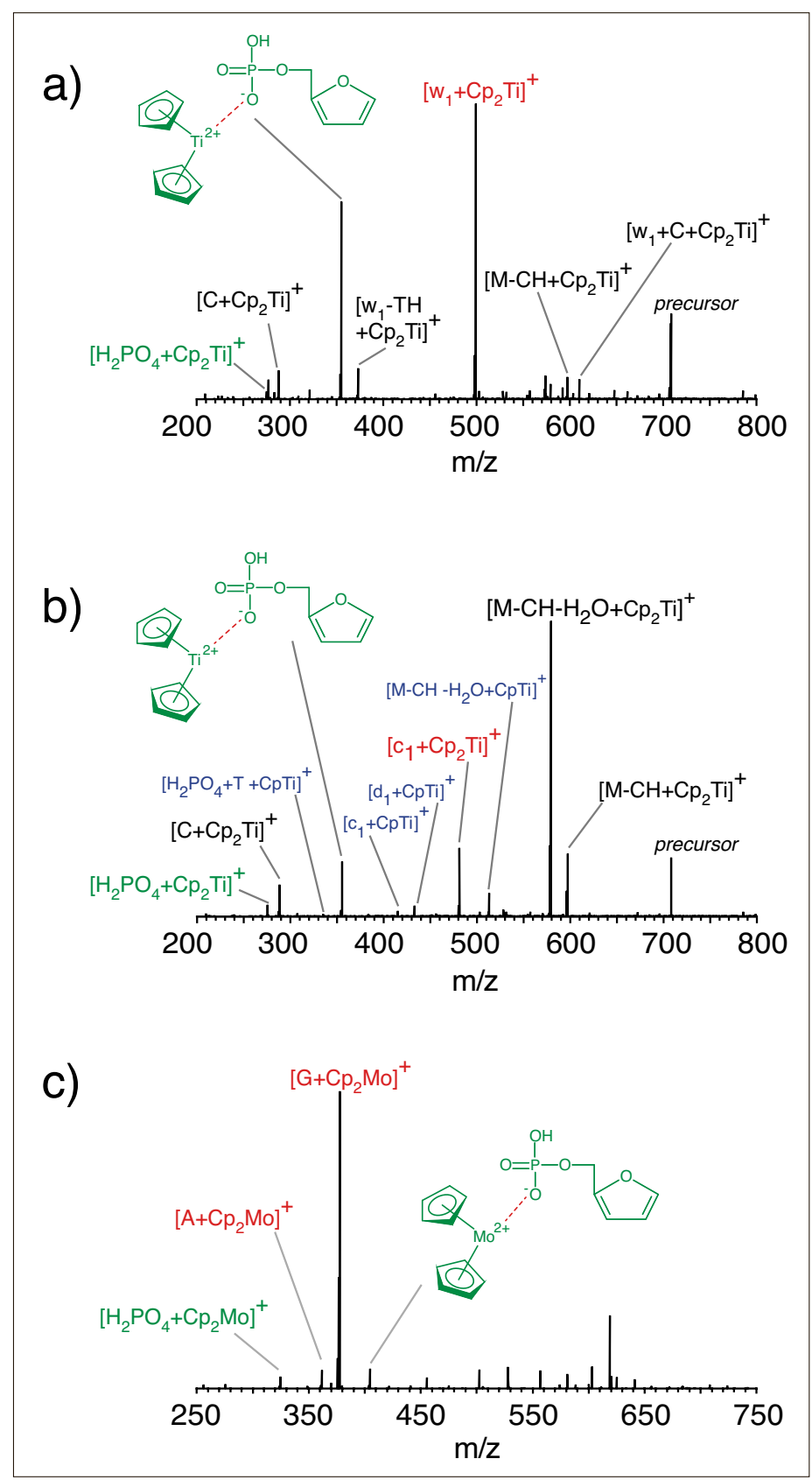

guanine nucleobase is much stronger than the interaction with the adjacent adenine, as reflected by the low peak intensity of $\left[\mathrm{A}+\mathrm{Cp}_{2} \mathrm{Mo}\right]^{+}$.

\section{Higher-order Nucleic Acids as Drug Targets}

Due to their important roles in cellular processes, higher-order structures, such as duplex- and quadruplex-DNA, represent potential drug targets as well. Despite the non-covalent binding characteristics of these assemblies, they do not necessarily separate upon electrospray ionization and collisional activation. Experiments on non-platinated and platinated quadruplexes revealed the formation of truncated structures due to the cleavage of covalent bonds in the oligonucleotide backbone without disrupting all non-covalent interactions between the nucleobases. ${ }^{[13,18]}$ This observation was somewhat unexpected, because low-energy CID is considered as a slow-heating ergodic process, which redistributes the added internal energy among the vibrational modes of the precursor ion prior to fragmentation and, therefore, was expected to result in the disruption of the non-covalent nucleobase interactions. In some cases, the detection of adducted and unadducted fragment ions allowed the localization of the adduct sites. Nevertheless, experimental alternatives are necessary when strand separation impedes precise characterization of the binding pattern.

\section{Alternative Ion Activation Techniques}

The various techniques used for ion activation in the gas-phase do not only differ in the amount of transferred energy, but also in the time frame required for the transfer of sufficient energy and in the mechanisms responsible for the dissociation of the precursor ions. The considerable long time frame required for low-energy collisional activation (up to $100 \mathrm{~ms}$ ) enables the redistribution of the added internal energy over the many degrees of freedom of the ions prior to fragmentation. This results in an ergodic dissociation behavior, slows down the reaction rate of covalent bond cleavage in large ions, and promotes the cleavage of non-covalent interactions, thus, impeding the investigation of higher-order structural features.

Alternative activation techniques that prevent energy randomization are applied to overcome this drawback. Among them are radical-based methods such as electron capture dissociation (ECD) and electron transfer dissociation (ETD). Transfer of electrons to a precursor ion population stored in a trapping instrument triggers oddelectron dissociation channels that provide sequence and structural information orthogonal to the data obtained by CID. The effect of various activation techniques on the types of generated fragment ions has been demonstrated for oligonucleotidecisplatin adducts. ${ }^{[12]}$ ETD was found to primarily result in charge reduction of the precursor ions, but backbone fragments could be generated by additional collisional activation of the charge-reduced precursor ions. The transfer of an electron to nucleic acids is highly nucleobase-specific with a high preference for abstraction by a cytosine nucleobase. ${ }^{[17,18]}$ Our recent ETD experiments on metallocene-DNA adducts of different charge states provided deeper insight into the radical-driven dissociation. Electron transfer to the doubly charged hexamer adduct resulted in charge reduction only. Interestingly, electron transfer to the triply charged precursor ion led to the doubly reduced adduct. The first electron was transferred to the metal center and the second one abstracted by the cytosine nucleobase, where it induced backbone cleavage (Fig. 3). The extent of electron transfer to the adduct was found to depend on the coordination center, which is likely to affect the dissociation mechanism. Elucidation of this mechanism represents the initial step on the way to extracting orthogonal structure information on higher-order nucleic acid assemblies and identifying the binding characteristics of metallodrugs. 


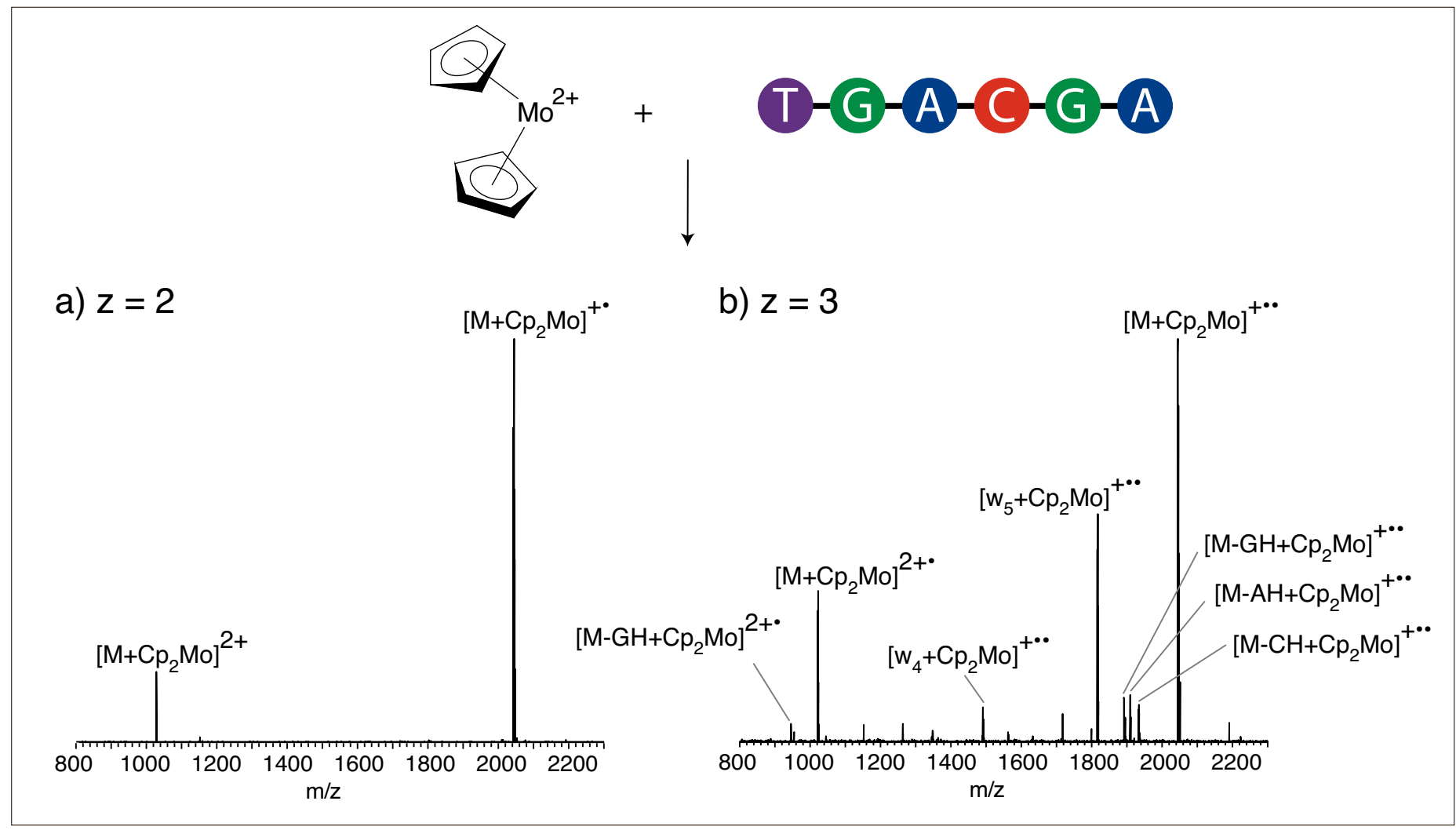

a) $z=2$

Fig. 3. Electron transfer dissociation of doubly and triply charged molybdenocene-DNA adducts.

\section{Conclusion}

The combination of accurate mass high-resolution mass spectrometry with multiple ion activation techniques forms a powerful toolkit for the investigation of biomolecule-drug interactions. Among the analytical techniques applied in the life sciences, tandem mass spectrometry excels with high sensitivity paired with the ability to isolate and activate precursor ions of interest in the gas-phase without the need for high sample purity. Knowledge on the dissociation behavior of the compound class of interest is an essential prerequisite for the reliable interpretation of the analytical data and, consequently, for the proper characterization of the biomoleculedrug adducts.
[1] Z. Wang, K. X. Wan, R. Ramanathan, J. S. Taylor, M. L. Gross, J. Am. Soc. Mass Spectrom. 1998, 9, 683.

[2] J. M. Tromp, S. Schürch, J. Am. Soc. Mass Spectrom. 2005, 16, 1262.

[3] J. Wu, S. A. McLuckey, Int. J. Mass Spectrom. 2004, 237, 197.

[4] S. T. M. Monn, S. Schürch, J. Am. Soc. Mass Spectrom. 2007, 18, 984.

[5] A. Nyakas, S. R. Stucki, S. Schürch, J. Am. Soc. Mass Spectrom. 2011, 22, 875.

[6] S. Schürch, Mass Spectrom. Rev. 2016, 35, 483.

[7] A. Nyakas, L. C. Blum, S. R. Stucki, J.L. Reymond, S. Schürch, J. Am. Soc. Mass Spectrom. 2013, 24, 249.

[8] M. Groessl, Y. O. Tsybin, C. G. Hartinger, B. K. Keppler, P. J. Dyson, J. Biol. Inorg. Chem. 2010, 15, 677.

[9] C. G. Hartinger, M. Groessl, S. M. Meier, A. Casini, P. J. Dyson, Chem. Soc. Rev. 2013, 42, 6186.
[10] A. M. Pizarro, A. Habtemariam, P. J. Sadler, Top. Organomet. Chem. 2010, 32, 21.

[11] A. Nyakas, M. Eymann, S. Schürch, J. Am. Soc. Mass Spectrom. 2009, 20, 792.

[12] Z. Xu, J. B. Shaw, J. S. Brodbelt, J. Am. Soc. Mass Spectrom. 2013, 24, 265.

[13] S. R. Stucki, A. Nyakas, S. Schürch, J. Mass Spectrom. 2011, 46, 1288.

[14] N. Kröger, U. R. Kleeberg, K. Mross, L. Edler, G. Sass, D. K. Hossfeld, Onkologie 2000, 23, 60.

[15] G. Lümmen, H. Sperling, H. Luboldt, T. Otto, H. Rübben, Cancer Chemother. Pharmacol. 1998, $42,415$.

[16] G. Kelter, N. J. Sweeney, K. Strohfeldt, H. H. Fiebig, M. Tacke, Anticancer Drugs 2005, 16, 1091.

[17] T. W. D. Chan, M. F. Choy, W. Y. K. Chan, Y. M. E. Fung, J. Am. Soc. Mass Spectrom. 2009, 20, 213.

[18] S. I. Smith, J. S. Brodbelt, Int. J. Mass Spectrom. 2009, 283, 85 . 